\title{
Contrasting time and frequency domains: ERN and induced theta oscillations differentially predict post-error behavior
}

\author{
Paul J. Beatty ${ }^{1} \cdot$ George A. Buzzell ${ }^{2} \cdot$ Daniel M. Roberts $^{1} \cdot$ Craig G. McDonald $^{1}$ \\ Published online: 17 April 2020 \\ (C) The Psychonomic Society, Inc. 2020
}

\begin{abstract}
The present study investigated the neural dynamics of error processing in both the time and frequency domains, as well as associated behavioral phenomena, at the single-trial level. We used a technique that enabled us to separately investigate the evoked and induced aspects of the EEG signal (Cohen \& Donner, 2013, Journal of Neurophysiology, 110[12], 2752-2763). We found that at the single-trial level, while the (evoked) error-related negativity (ERN) predicted only post-error slowing (PES)and only when errors occurred on incongruent trials - induced frontal midline theta power served as a robust predictor of both PES and post-error accuracy (PEA) regardless of stimulus congruency. Mediation models of both electrophysiological indices demonstrated that although the relationship between theta and PEA was mediated by PES, there was not a relationship between the ERN and PEA. Our data suggest that although the ERN and frontal midline theta index functionally related underlying cognitive processes, they are not simply the same process manifested in different domains. In addition, our findings are consistent with the adaptive theory of post-error slowing, as PES was positively associated with post-error accuracy at the single-trial level. More generally, our study provides additional support for the inclusion of a time-frequency approach to better understand the role of medial frontal cortex in action monitoring.
\end{abstract}

Keywords ERN $\cdot$ Frontal midline theta $\cdot$ Action monitoring $\cdot$ Induced vs. evoked

Within simple forced-choice decision-making tasks, committing an error initiates a cascade of cognitive events within the action-monitoring system. The participant will briefly disengage from the ongoing task, attend to and identify the error, and make cognitive and behavioral adjustments in an effort to prevent the error from reoccurring once they reengage in the task (Wessel, 2017; Wessel \& Aron, 2017). In particular, these behavioral adjustments include increases in response time on trials that follow error commission (post-error slowing [PES]; Laming, 1979), as well as either increases or decreases in subsequent trial accuracy (post-error accuracy [PEA]). A major topic of debate in the research community is whether this

Electronic supplementary material The online version of this article (https://doi.org/10.3758/s13415-020-00792-7) contains supplementary material, which is available to authorized users.

Paul J. Beatty

pbeatty@masonlive.gmu.edu

1 George Mason University MS 3F5, 4400 University Drive, Fairfax, VA 22030, USA

2 Department of Psychology at University of Maryland, College Park, MD 20742, USA slowing is an adaptive or maladaptive consequence of an error. Findings in support of adaptive theories suggest that PES provides additional time to process subsequent trial task stimuli, consequently enabling a relative improvement in task performance (increased PEA; Botvinick, Braver, Barch, Carter, \& Cohen, 2001; King, Korb, von Cramon, \& Ullsperger, 2010; Maier, Yeung, \& Steinhauser, 2011; Marco-Pallarés, Camara, Münte, \& Rodríguez-Fornells, 2008; M. Steinhauser \& Andersen, 2019). By contrast, findings in support for maladaptive theories suggest that PES is an index of distraction from the task set that leads to a deterioration of performance and potentially additional errors (decreased PEA; Beatty, Buzzell, Roberts, \& McDonald, 2018; Buzzell, Beatty, Paquette, Roberts, \& McDonald, 2017; Jentzsch \& Dudschig, 2009; Notebaert et al., 2009; R. Steinhauser, Wirth, Kunde, Janczyk, \& Steinhauser, 2018; van den Brink, Wynn, \& Nieuwenhuis, 2014; Van der Borght, Schevernels, Burle, \& Notebaert, 2016). Despite attempts to reconcile this debate (Purcell \& Kiani, 2016; Ullsperger \& Danielmeier, 2016), the research community has not yet found consensus.

To inform the link between error processing and how individuals adjust their behavior following error commission, researchers have identified various neural indices of error 
processing. The error related negativity (ERN; Falkenstein, Hohnsbein, Hoormann, \& Blanke, 1991; Gehring, Goss, Coles, Meyer, \& Donchin, 1993) is a response-locked, frontocentral event-related potential (ERP) component generated at least partially within the medial frontal cortex (Buzzell, Richards, et al., 2017) that is believed to act as an implicit signal to convey either a mismatch between expected and actual performance (Gehring et al., 1993) or response conflict associated with competing responses (Botvinick et al., 2001). Studies of the relation between the ERN and post-error behavioral adaptations have yielded conflicting results. Whereas some work has shown the ERN to be associated with increased PES (Debener et al., 2005; Fischer, Danielmeier, Villringer, Klein, \& Ullsperger, 2016; Fu et al., 2019; Gehring et al., 1993; Kalfaoğlu, Stafford, \& Milne, 2018; Maier et al., 2011; M. Steinhauser \& Andersen, 2019), other studies have failed to find any relation between the ERN and post-error behavior (Beatty et al., 2018; Buzzell, Beatty, et al., 2017; Dudschig \& Jentzsch, 2009; Nieuwenhuis, Ridderinkhof, Blom, Band, \& Kok, 2001; Valadez \& Simons, 2017; Van der Borght et al., 2016). While a recent meta-analysis provided support for a relation between the ERN and PES (Cavanagh \& Shackman, 2015), this study did not investigate possible relations with PEA. To our knowledge, even in cases in which the ERN predicts PES, it has not directly predicted increased PEA, which has made it difficult to draw conclusions as to whether neural activity associated with PES is adaptive or maladaptive.

In addition to the ERN, research has also been conducted on transient, rhythmic cortical activity, which is believed to originate from the medial frontal cortex (Cohen, 2014). In particular, frontal midline theta oscillations $(4-7 \mathrm{~Hz})$ that occur around the time of response are thought to act as an implicit monitoring signal (Ullsperger, Fischer, Nigbur, \& Endrass, 2014) that leads to the recruitment of cognitive control and post-error compensations (Cavanagh \& Frank, 2014). A recent study conducted by Valadez and Simons (2017) investigated differences in the predictive power that ERPs (such as the ERN) and neural oscillations (such as frontal midline theta) have for post-error behavior. They determined that, although the ERN did not have a relationship with post-error behavior, the magnitude of theta oscillations was related to increases in both PES and PEA. They interpreted their findings as providing support for the adaptive theory of PES, which posits that slowing down after an error reflects enhanced cognitive control, which consequently leads to performance recovery.

Although Valadez and Simons (2017) provided compelling evidence that ERP and time-frequency analyses differ in their ability to predict post-error behavioral compensations, it is still controversial as to whether the ERN and theta oscillations reflect either the same neural process manifested in different domains or dissociable elements of the error-monitoring system (Luu \& Tucker, 2001; Luu, Tucker, \& Makeig, 2004;
Yeung, Bogacz, Holroyd, \& Cohen, 2004; Yeung, Bogacz, Holroyd, Nieuwenhuis, \& Cohen, 2007). Indicative of the widespread assumption that the ERN, or other controlsensitive frontal-midline ERPs (i.e., the ERN, N2, and FRN), are relatively synonymous with theta oscillations assessed via time-frequency approaches, a recent metaanalysis of "frontal midline theta" (Cavanagh \& Shackman, 2015) relied primarily on ERPs as a means to study relations between "theta" and behavior. Thus, additional work investigating the relations between theta, the ERN, and post-error behavior are needed. Since the theta power analyses presented by Valadez and Simons (2017) included both the evoked (phase-locked) and induced (non-phase-locked) components of the EEG signal, they were not able to rule out that the predictive power of theta was due to the residual influence of the evoked data. Here, we note that one study in particular has directly investigated how the evoked and induced portions of the event-related theta power can be dissociated, and how these neural elements relate to behavior. Cohen and Donner (2013) pioneered a technique in which the average evoked component of the neural response (the ERP) is subtracted from the total EEG signal before computing the time-frequency power spectrum, allowing for separate investigation of both evoked and induced neural activity. Using this technique, they were able to dissociate the conflict-related N2 from induced theta oscillations and observed that only induced theta power was predictive of conflict-related response slowing. Given these findings, we chose to use Cohen and Donner's (2013) approach to investigate whether the ERN and frontal midline theta could be similarly dissociated, and if so, exhibit differential relations with post-error behavior. We note however, that although Cohen and Donner (2013) demonstrated that induced theta was a better predictor of next-trial RT slowing, since they did not investigate the relationship between induced theta and next-trial accuracy, they could not conclude whether this RT slowing was adaptive or maladaptive.

Valadez and Simons (2017) provided seminal evidence for a relationship between frontal midline theta oscillations and performance recovery (increased accuracy); they also provided support for the notion that PES is adaptive. However, their analysis approach did not leverage trial-by-trial relations between brain and behavior and instead relied on partitioning task data into trial bins, leading to a rather small number of trials per condition and the exclusion a majority of their participants for some analyses. Furthermore, Valadez and Simons (2017) did not attempt to dissociate the induced and evoked components of the EEG signal. Given these considerations, we sought to build upon the findings of Valadez and Simons (2017) using a single-trial analysis and an approach that enabled us to dissociate evoked and induced neural activity (Cohen \& Donner, 2013). Based on the findings of Valadez and Simons (2017), we predicted that induced theta oscillations, but not the evoked neural activity of the 
ERN, would predict post-error behavior (PES and PEA) at the single-trial level. Moreover, we predicted that PES would be positively associated with PEA, in line with adaptive theories of PES.

\section{Method}

The present study uses the same paradigm and data as reported in Beatty et al. (2018); for completeness, the general procedures are outlined below.

\section{Participants and experiment design}

Twenty-four healthy, right-handed participants (mean age $=$ 24.75 years; 16 female) participated in exchange for course credit at George Mason University. Three participants were excluded due to either below-chance accuracy, routinely correcting responses (more than two-thirds of errors were corrected), or procedural error during data collection, leaving 21 participants (mean age $=23.62$ years; 13 female) to be incorporated into all analyses. Participants completed a color Simon task (see Fig. 1), implemented using Psychtoolbox (Brainard, 1997; Kleiner et al., 2007; Pelli, 1997) for the MATLAB programming environment (The MathWorks, Natick, MA) in conjunction with custom scripts and functions. Participants were presented with a light-gray central fixation cross on a darker gray background along with two light-gray boxes, each subtending $3.75 \times 3.75$ degrees, located 4.25 degrees to the left and right of the center of the screen. A red (sRGB: $r=105.85, g=0, b=0$ ) or green (sRGB: $r=0, g=$ $53.05, \mathrm{~b}=0$ ) colored circle, subtending 2 degrees, was presented for $200 \mathrm{~ms}$ in the center of one of the two boxes. All stimuli were equiprobable, and perceived luminance of the stimuli was equated using the sRGB gamma function. The task was presented on the Cambridge Research Systems Display++ LCD monitor (Cambridge Research Systems Ltd).

Participants were instructed to respond to each stimulus as quickly and accurately as possible by pressing either the " 2 " key (using their left index finger) or the " 8 " key (using their right index finger) to indicate what color was presented. These response mappings were counterbalanced across participants. If the participant responded within $150 \mathrm{~ms}$ of stimulus onset, responded after the 500-ms response deadline, corrected their response, or did not provide a response, the trial (as well as the following trial) were removed from all analyses. Following the response, participants were provided with a randomly selected response stimulus interval (RSI) from a random uniform distribution between $200 \mathrm{~ms}$ and 1,200 $\mathrm{ms}$ in duration. In order to combat fatigue, between each block participants were required to rest for at least 30 seconds prior to beginning the next block. During this period, they were also informed of their block accuracy. The main experiment consisted of 3,520 trials (22 blocks of 160 trials). The last two blocks, however, were dedicated to passive viewing of the stimuli and are not discussed here.

To familiarize themselves with the task, participants first completed an easier version, in which they were provided with a 2-second response deadline as well as trial-by-trial feedback of their performance (no trial-level accuracy feedback was presented during the actual experiment). Following practice of an easy version of the task, participants completed an additional practice that was more difficult and identical to the experimental version of the task.

\section{EEG data acquisition and processing}

EEG data were collected using a Brain Vision ActiChamp amplifier and Brain Vision Recorder 1.2 acquisition software (Brain Products Inc.). Data were recorded using 64 actiCAP electrodes (positioned according to the extended 10-20 system). An additional in-cap ground electrode was positioned anterior to electrode Fz. Although electrode $\mathrm{Cz}$ was used as the online reference for the other 63 in-cap electrodes, the data for electrode $\mathrm{Cz}$ were recovered off-line. The data were then re-referenced off-line to the average of electrodes TP9 and TP10 (corresponding to the left and right mastoids). The data were recorded at a sampling rate of $1,000 \mathrm{~Hz}$, with an online

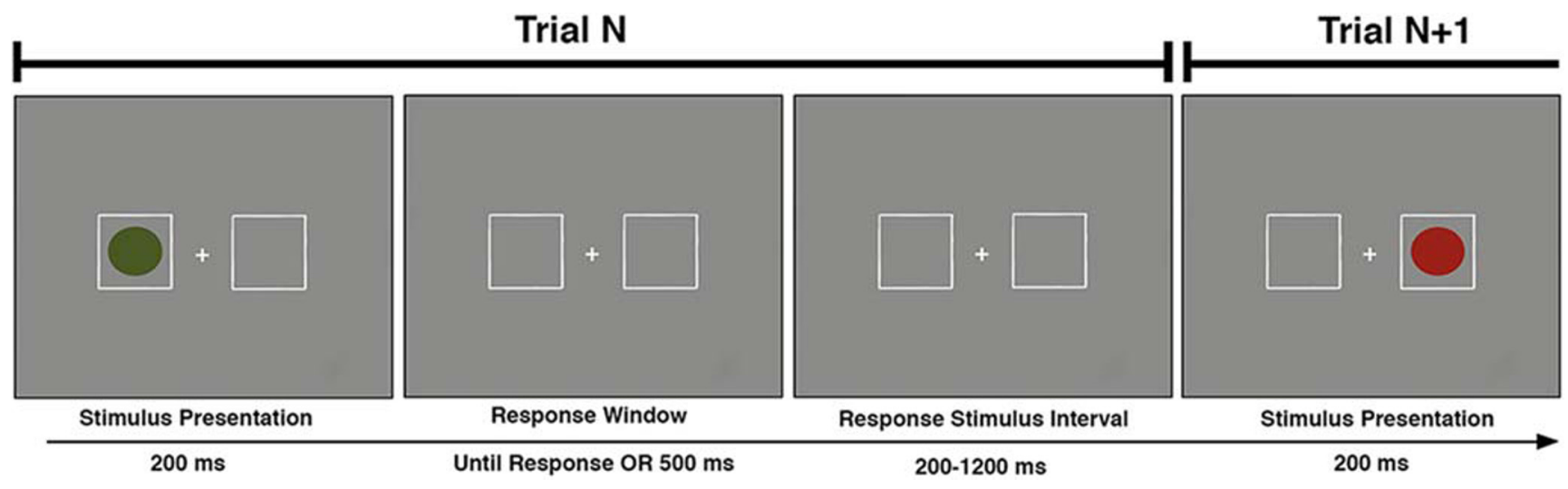

Fig. 1 Experimental paradigm. Stimulus contrasts have been increased for presentation purposes 
band-pass filter of .1-250 Hz. In concordance with impedance recommendations for high-impedance active EEG systems, impedance for all electrodes was maintained below $25 \mathrm{k} \Omega$.

Processing of EEG data was conducted using the EEGLAB (Delorme \& Makeig, 2004) toolbox for the MATLAB programming environment (The MathWorks, Natick, MA). Data were detrended, low-pass filtered at $30 \mathrm{~Hz}$ (using a Butterworth filter from the ERPLAB plugin; LopezCalderon \& Luck, 2014) and down-sampled to $500 \mathrm{~Hz}$. On a 1-Hz high-pass filtered copy of the original data set (which was preprocessed in an identical manner to the $0.1-\mathrm{Hz}$ highpass filtered data set), the data were segmented into a series of consecutive 1,000-ms epochs and subjected to automated amplitude rejection threshold of $\pm 500 \mu \mathrm{V}$ and a spectral rejection threshold of $50 \mathrm{~dB}$ within the $20-40 \mathrm{~Hz}$ band using the pop_rejspec function (to remove EMG-like activity; Delorme \& Makeig, 2004). If more than $20 \%$ of epochs were marked for rejection in a given channel, that channel was removed from all copies of the data set. The data were then decomposed using independent component analysis (ICA). The ICA component weights that were generated for the 1$\mathrm{Hz}$ high-pass filtered data set were then copied onto the original 0.1-Hz high-pass data set (Winkler, Debener, Müller, \& Tangermann, 2015). All further analyses were performed on the $0.1-\mathrm{Hz}$ high-pass data set.

After rejecting independent components that corresponded to blinks and saccades, the data were epoched from $-1,000 \mathrm{~ms}$ to $1,500 \mathrm{~ms}$ for all stimulus and response markers. To remove epochs containing residual artifacts after cleaning with ICA, the data were subjected to a stricter automated rejection procedure using a voltage rejection threshold of $\pm 100 \mu \mathrm{V}$ and a spectral rejection threshold of $50 \mathrm{~dB}$ within the $20-40 \mathrm{~Hz}$ band using the pop_rejspec function. Similar to earlier in the processing stream, if the more than $20 \%$ of epochs were marked for rejection for a given channel, that channel was removed from the data set. In order to ensure that the trial $N$ stimulus, trial $N$ response, and trial $N+1$ stimulus epochs were matched, if any member of the epoch "triplet" were rejected during processing, the corresponding epochs were also rejected. All rejected channels were then interpolated (using a spherical spline interpolation). If the maximum number of channels that were interpolated for a participant was more than $10 \%$ of all electrodes, that participant was removed from all analyses. To better estimate cortical sources and reduce the impact of volume conduction, we conducted a Laplacian (current source density [CSD]) transformation of the data (Kayser \& Tenke, 2006).

When conducting time-frequency analyses, we used the approach taken by Cohen and Donner (2013) to remove the ERP from the EEG signal. With this approach, the averaged ERPs are subtracted from each individual epoch before computing the time-frequency spectrum, thus theoretically removing the phase-locked component of the EEG signal. This assumes that the ERP components are relatively timeinvariant from trial to trial. This subtraction was performed in the time domain (using the full $-1,000-\mathrm{ms}$ to $1,500-\mathrm{ms}$ epoch) and was completed separately at each electrode for each condition of interest. Although only the induced neural activity was subjected to statistical analysis, time-frequency analyses were separately performed on both the total and induced versions of the data set in order to demonstrate that the method provided results comparable to those of Cohen and Donner (2013; see Fig. 2). Note that the total and induced plots are very similar, which suggests that the method did not dramatically alter the data in which there was comparatively little influence of the evoked activity within the theta frequency range. It is to be expected that the subtraction does not dramatically change the time-frequency power distribution since the majority of power in time-frequency data comes from non-phase-locked (induced) activity (Trujillo \& Allen, 2007); the primary difference is that activity in the delta frequency range was removed. Consistent with these observations, the intertrial phase coherence (ITPC) was relatively higher within the delta band, as compared with the theta band, which suggests that power in this frequency range reflects proportionately greater phase-locked activity.

Following subtraction of the ERP, the data were transformed to a time-frequency representation via convolution with a series of complex Morlet wavelets at the single-trial level. The frequency of each wavelet increased from 2 to $30 \mathrm{~Hz}$ in 20 linearly spaced steps, while the number of cycles increased from 3 to 6 in 20 logarithmically spaced steps. We note that the complete data set contained trials with RSIs ranging from $200 \mathrm{~ms}$ to $1,200 \mathrm{~ms}$ (for the purposes of orthogonal analyses described in Beatty et al., 2018). In order to ensure that a sufficient baseline normalization period was used for the full RSI range, the data were binned into three tertiles (short: 200-533 ms; medium: 534-866 ms; long: 8671,200 ms). Given the limited time between trials for short RSI, in an attempt to avoid overlap from the previous trial inside the baseline period, we elected to baseline normalize the short RSI bin using the same baseline that we used for medium and long RSIs. More specifically, the power values at each frequency were baseline normalized using a common, divisive baseline relative to the average power from $-400 \mathrm{~ms}$ to $-100 \mathrm{~ms}$ relative to stimulus onset, collapsing across medium/long RSIs and all conditions of interest $\left(10 \times \log _{10}\right.$ (trial $N$ response power / common trial $N$ stimulus baseline period)).

For all analyses, PES was computed at the average (group) and single-trial level by subtracting current trial error RTs from next trial post-error RTs (Schroder et al., 2019), while PEA was computed as a percentage change between (1) the accuracy for trials following an error and (2) the accuracy for trials following a correct response (Beatty et al., 2018; Buzzell, Beatty, et al., 2017). To verify whether there was a 
significant magnitude of slowing at each RSI bin, we conducted a series of Bonferroni-corrected $t$ tests. Analyses of PES and PEA at the group level were separately conducted using a 2 (congruency: congruent, incongruent) $\times 3$ (RSI: short, medium, long) analysis of variance (ANOVA).

For ERP analyses, given that all ERP analyses were conducted using the same trials that were used in the timefrequency analyses, the trial $N$ stimulus and response epochs were reepoched to $-200 \mathrm{~ms}$ to $800 \mathrm{~ms}$. Baseline correction was implemented using the prestimulus period $(-200 \mathrm{~ms}$ to 0 $\mathrm{ms}$ ) from the trial-matched stimulus-locked epoch. We observed that artifact rejection affected error trials $(24.133 \%$ rejected) to a greater extent than correct trials $(13.649 \%$ rejected), $\mathrm{t}(20)=-5.214, p<.001$; this is perhaps due to additional ocular or muscular artifacts that occurred in concordance with an erroneous response. Nevertheless, we still had a sufficient number of trials per condition (Olvet \& Hajcak, 2009). The average number of clean, artifact-free trials incorporated into each condition of interest were as follows: "correct congruent followed by correct" $(M=853.95, S D=$ 147.66), "error congruent followed by correct" $(M=97.00$, $S D=40.22)$, "correct incongruent followed by correct" $(M=$ $774.67, S D=136.51$ ), "error incongruent followed by correct" $(M=158.71, S D=60.37)$. Statistical analyses for the ERN and theta at the group level took place at electrode $\mathrm{Cz}$ (which is where both components were found to be maximal) using separate 2 (accuracy: correct, error) $\times 2$ (congruency: congruent, incongruent) ANOVAs. The values being compared were the average voltage (for the ERN) or average power (for theta) within their respective analysis windows, centered at the peak of each component at the group level. The "peak" of theta was chosen by plotting theta power as a timeseries plot (averaging across the data derived from wavelets between 4 to $7 \mathrm{~Hz}$ ). Analysis windows were constructed on an individual participant basis in order to better capture each component at the single-trial level. To create analysis window widths that were data driven, we elected to create a windowwidth based on the $50 \%$ amplitude of the component (Hassall, McDonald, \& Krigolson, 2019). The averaged window width across participants was computed to be from approximately
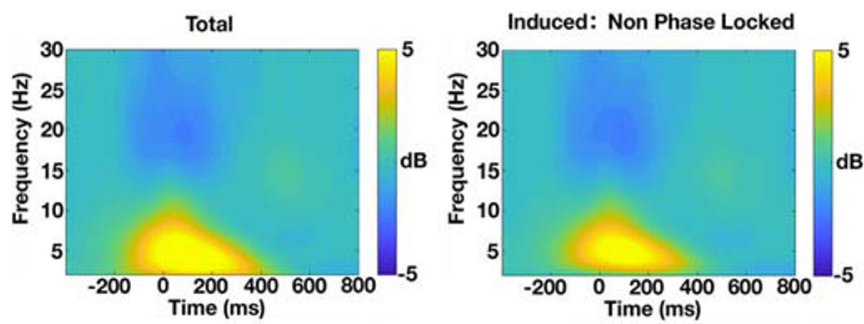

Fig. 2 Separation of evoked and induced power. Similar to the findings of Cohen and Donner (2013), these plots demonstrate how total power can be parsed into induced and evoked power. The "Evoked" plot was computed by subtracting the ERSP values of "Induced" from "Total." Note that the total and induced plots are very similar, which suggests that the
$-6 \mathrm{~ms}$ to $78 \mathrm{~ms}$ for the ERN and $-110 \mathrm{~ms}$ to $258 \mathrm{~ms}$ for theta power.

To assess whether error processing (as indexed by the ERN and theta) was predictive of changes in subsequent trial behavioral indices of cognitive control on a single-trial (withinsubject) level, using the same electrode and windows as in the group-level analyses, we performed a series of mixed-effects models using the R statistical software (Version 3.3.1; Imai, Keele and Tingley, 2010), using the lmerTest package (Version 2.0-32; Kuznetsova, Brockhoff, \& Christensen, 2016) and the lme4 package (Version 1.1-12; Bates, Mächler, Bolker, \& Walker, 2014). Prior to carrying out each analysis, models were constructed such that the continuous variables (ERN magnitude, theta magnitude, PES, RSI) were centered and scaled to have a mean of zero and standard deviation of 1. Instead of keeping RSI segmented into bins, we elected to have RSI as a continuous variable for all relevant single-trial models. These variables were fit using linear mixed-effects analysis using the lmer function, with restricted maximum likelihood estimation. The categorical variable (PEA) was examined using sum contrasts and fit using generalized linear mixedeffects models using the glmer function with logit link with maximum likelihood estimation. Variation in intercept was treated as a random effect, while all remaining variables were treated as fixed effects. Statistical significance for each fixed effect was calculated via lmerTest (Kuznetsova et al., 2016), using the Satterthwaite's approximation to denominator degrees of freedom. The main analyses explored the effect of ERP component magnitude or theta magnitude predicting singletrial modulation of post-error behavior (PES and PEA). In order to determine whether theta or the ERN accounts for more variance when predicting subsequent trial behavior, an additional model was conducted with both indices in the same model. Since we were interested specifically in error processing, we limited what types of trials would be included in the models. When predicting PES, we limited the trials that were included in the model to "error trials followed by correct trials." When predicting PEA, we limited the trials such that the current trial must be an error. Furthermore, to follow up on significant effects in the mixed-effects models, we conducted separate
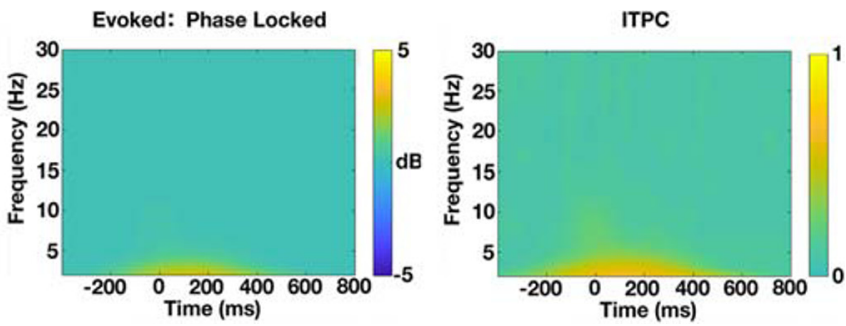

method did not dramatically alter the data. The assumption being made during the subtraction process is that the ERP components are relatively time invariant from trial to trial. The intertrial phase coherence (ITPC) plot illustrates the magnitude of response-locked phase locking (mean vector length). (Color figure online) 
mediation models for induced theta power and the ERN using the mediation package for R (Tingley, Yamamoto, Hirose, Keele, \& Imai, 2014) to determine whether each indices' predictive power for PEA was mediated by PES. As recommended by the mediation package, the models underwent 1,000 iterations, which were sampled using a quasi-Bayesian Monte Carlo method based on normal approximation (Imai, Keele, \& Tingley, 2010).

Since a change in statistical significance between ERN and theta models cannot alone be used to infer that one model provides a better fit to behavioral data (Gelman \& Stern, 2006; Nieuwenhuis, Forstmann, \& Wagenmakers, 2011), the fit of ERN and theta models were directly compared using Akaike weights (Burnham \& Anderson, 2003; Wagenmakers \& Farrell, 2004). Specifically, the secondorder Akaike information criterion (AICc) was calculated for both ERN and theta models using the "AICc" function in the MuMIn package (Barton, 2019) and subsequently transformed to Akaike weights. The probability that the best fitting model was indeed the preferred model was calculated as the ratio of the best fitting model Akaike weights to the sum of Akaike weights from all models under consideration (both ERN and theta).

\section{Results}

\section{Group-level analyses}

\section{Post-error behavior}

A series of Bonferroni-corrected $t$ tests regarding the magnitude of PES for each RSI tertile revealed that there indeed was significant slowing for all three RSI bins: short (200-533 ms), $t(20)=8.189, p<.001$; medium $(534-866 \mathrm{~ms}), t(20)=5.949$, $p<.001$; long $(867-1,200 \mathrm{~ms}), t(20)=5.122, p<.001$.

For analyses of PES, a 2 (congruency: congruent, incongruent) $\times 3$ (RSI: short, medium, long) ANOVA revealed an effect of congruency on PES, $F(1,20)=84.810, p<.001, \eta_{\mathrm{p}}{ }^{2}$ $=.809$, in which slowing following incongruent trials $(M=$ $36.751 \mathrm{~ms}, S D=3.683 \mathrm{~ms}$ ) was greater than following congruent trials $(M=11.640 \mathrm{~ms}, S D=3.684 \mathrm{~ms})$, as well as an effect of RSI on PES, $F(1,20)=26.618, p<.001, \eta_{\mathrm{p}}{ }^{2}=.571$, in which slowing following short RSI $(M=38.262 \mathrm{~ms}, S D=$ $4.710 \mathrm{~ms})$ was greater than following medium $(M=16.939$ $\mathrm{ms}, S D=3.500 \mathrm{~ms})$ or long $(M=17.386 \mathrm{~ms}, S D=3.442 \mathrm{~ms})$ RSI. However, the congruency by RSI interaction failed to reach significance $(p=.431)$.

For analyses of PEA, a 2 (congruency: congruent, incongruent) $\times 3$ (RSI: short, medium, long) ANOVA revealed an effect of congruency on PEA, $F(1,20)=6.293, p=.021, \eta_{\mathrm{p}}{ }^{2}=$ .239 , in which accuracy following incongruent errors $(M=$ $-9.800 \%, S D=-2.000 \%$ ) was lower than following congruent errors $(M=-6.700 \%, S D=2.200 \%)$, as well as an effect of RSI on PEA, $F(1,20)=25.207, p<.001, \eta_{\mathrm{p}}{ }^{2}=$ .558 , in which accuracy following short RSI $(M=-13.800 \%$, $S D=2.200 \%)$ was lower than following medium $(M=$ $-6.800 \%, S D=2.300 \%)$ or long $(M=-4.100 \%, S D=$ $2.000 \%)$ RSI. However, the congruency by RSI interaction failed to reach significance $(p=.914)$.

\section{Electrophysiology}

Analysis of the ERN (see Fig. 3a) revealed a main effect of accuracy, $F(1,20)=43.636, p<.001, \eta_{\mathrm{p}}{ }^{2}=.686$, in which the ERN $(M=-21.679, S E=2.915)$ was larger than the correctrelated negativity $(\mathrm{CRN})(M=-.706, S E=2.313)$, as well as a main effect of congruency, $F(1,20)=4.962, p=.038, \eta_{\mathrm{p}}{ }^{2}=$ .199 , such that the ERN on incongruent trials $(M=-11.811$, $S E=2.062)$ was larger than on congruent trials $(M=-10.574$, $S E=2.171)$. There was also an Accuracy $\times$ Congruency interaction, $F(1,20)=7.679, p=.012, \eta_{\mathrm{p}}{ }^{2}=.277$. Pairedsamples $t$ tests revealed that the CRN on congruent trials ( $M$ $=-1.043, S E=2.309)$ was not significantly different than on incongruent trials $(M=-.369, S E=2.346 ; p=.211)$, but the ERN on incongruent trials $(M=-23.252, S E=3.038)$ was larger than on congruent trials $(M=-20.106, S E=2.901 ; p=$ $.012, d=.229$ ).

Analysis of theta power (see Fig. 3b) revealed a main effect of accuracy, $F(1,20)=200.269, p<.001, \eta_{\mathrm{p}}{ }^{2}=.909$, in which theta power for errors $(M=4.349, S E=.290)$ was larger than theta power for corrects $(M=1.471, S E=.169)$, but failed to reveal a main effect of congruency $(p=.934)$. However, there was also an Accuracy $\times$ Congruency interaction, $F(1,20)=$ $19.514, p<.001, \eta_{\mathrm{p}}{ }^{2}=.494$. Paired-samples $t$ tests revealed that theta power on incongruent trials $(M=1.633, S E=.184)$ was larger than on congruent trials $(M=1.309, S E=.160)$ for corrects $(p<.001, d=.383)$, but the theta power on congruent trials $(M=4.517, S E=.331)$ was larger than on incongruent trials $(M=4.181, S E=.260)$ for errors $(p=.019, d=.210)$.

The analyses for both the group-level ERN and induced theta (1) verified that activity on error trials is larger than activity on correct trials and (2) determined that, although in opposite directions, both the ERN and theta were modulated by congruency on error trials.

\section{Single-trial analyses}

In order to investigate the ERN and theta at the singletrial level, we conducted a series of mixed effects models (see Fig. 4).

\section{Predicting PES}

For the model of "theta, congruency, and RSI predicting PES" $(\mathrm{AICc}=14,912.43)$, there was an effect of theta, $t(5197)=$ 
2.509 , estimate $=.035, S E=.014, p=.012$, in which larger theta magnitude predicted slower responding on the following trial. However, the Theta $\times$ Congruency interaction, $t(5353)=$ -.017 , estimate $=-.0004, S E=.027, p=.987$, Theta $\times$ RSI interaction, $t(5345)=-.571$, estimate $=-.008, S E=.013, p=$ .568 , and Theta $\times$ Congruency $\times$ RSI interaction, $t(5348)=$ -.825 , estimate $=-.022, S E=.027, p=.410$, failed to reach significance. For the model of "ERN, congruency, and RSI predicting PES" $(\mathrm{AICc}=14,911.45)$, the effect of ERN, $t(5201)=-.357$, estimate $=-.005, S E=.014, p=.721$, ERN $\times$ RSI interaction, $t(5348)=-1.367$, estimate $=-.019$, $S E=.014, p=.172$, and ERN $\times$ Congruency $\times$ RSI interaction, $t(5346)=-.744$, estimate $=-.021, S E=.028, p=.457$, failed to reach significance. However, there was a significant ERN $\times$ Congruency interaction, $t(5351)=-2.145$, estimate $=$ $-.058, S E=.027, p=.032$. Follow-up statistical models demonstrated that a larger ERN led to slower responding following incongruent, $t(3138)=-1.959$, estimate $=-.035, S E=$ $.018, p=.050$, but not congruent, $t(1960)=.955$, estimate $=$ $.022, S E=.023, p=.340$, trials. For the model of "theta, ERN, congruency, and RSI predicting PES," there was an effect of theta, $t(5247)=2.299$, estimate $=.033, S E=.014, p=.022$, in which larger theta magnitude predicted slower responding on the following trial. All other effects regarding theta or ERN failed to reach significance. Therefore, theta explained unique variance in terms of predicting PES, over and above any effect of the ERN.

\section{Predicting PEA}

For the model of "theta, congruency, and RSI predicting PEA" (AICc $=7,452.066)$, there was an effect of theta $(z=4.613$, estimate $=.141, S E=.030, p<.001)$, in which larger theta magnitude predicted increased accuracy on the following trial. However, the Theta $\times$ Congruency interaction $(z=-.183$, estimate $=-.011, S E=.058, p=.855)$, Theta $\times$ RSI interaction $(z=-.157$, estimate $=-.005, S E=.029, p=.875)$, and Theta $\times$ Congruency $\times$ RSI interaction $(z=.762$, estimate $=.044, S E=$ $.058, p=.446)$, failed to reach significance. For the model of "ERN, congruency, and RSI predicting PEA" $(\mathrm{AICc}=$ $7,466.837)$, although the effect of ERN $(z=-1.551$, estimate $=-.049, S E=.032, p=.121)$, the ERN $\times$ Congruency inter action $(z=1.235$, estimate $=.075, S E=.061, p=.217)$, and $\mathrm{ERN} \times$ Congruency $\times \mathrm{RSI}$ interaction $(z=-.713$, estimate $=$ $-.044, S E=.062, p=.476$ ), failed to reach significance, there was a trend for the ERN $\times$ RSI interaction $(z=1.897$, estimate $=.059, S E=.031, p=.058)$. For the model of "theta, ERN, congruency, and RSI predicting PEA," there was an effect of
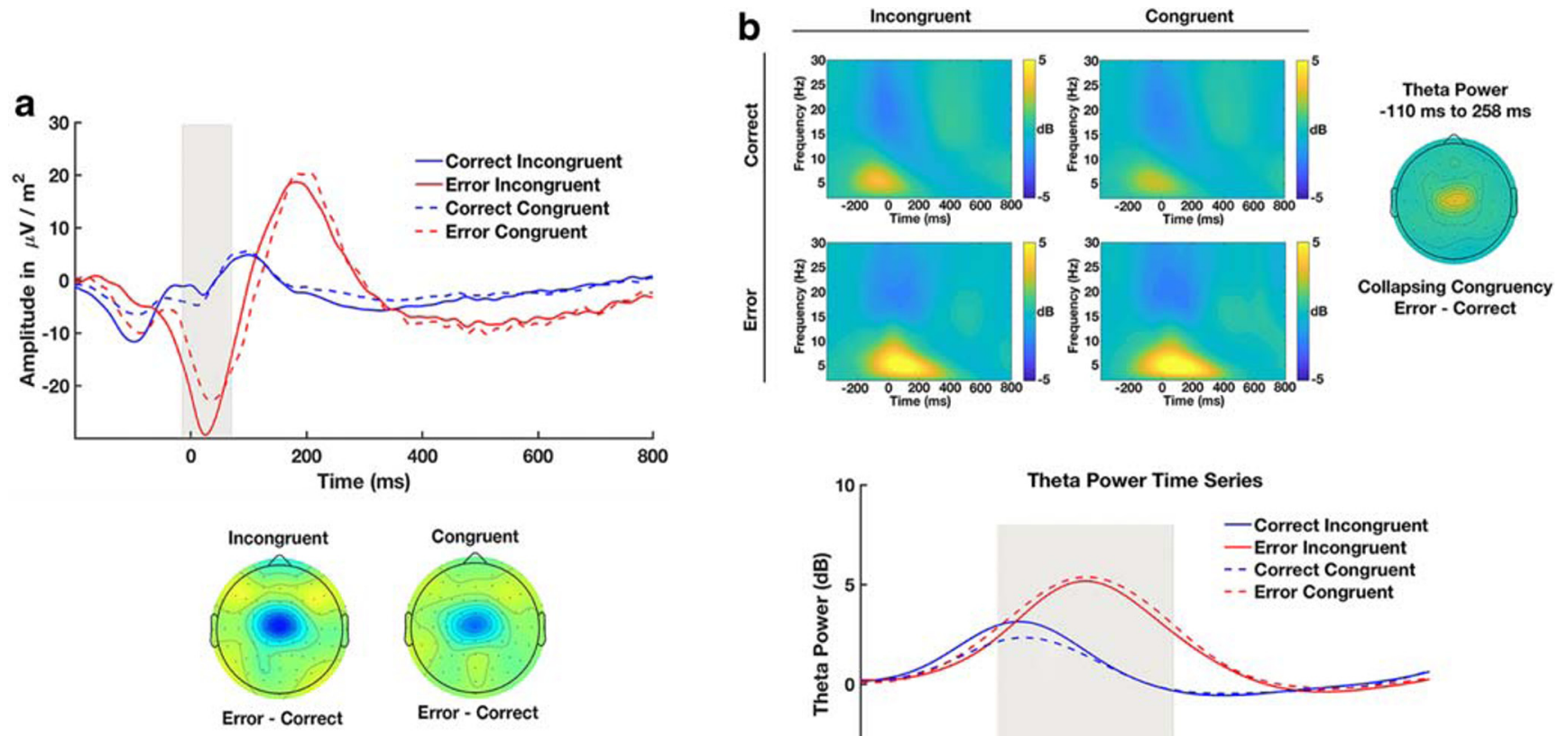

Fig. 3 ERN and theta analyses. Although the analysis windows were constructed on an individual person basis using a 50\% amplitude measure, the average analysis window (collapsed across participants) is designated by the shaded box. a ERN at electrode $\mathrm{Cz}$ (collapsed across RSI) with topographic plots as a function of congruency. The topographic plots depict the amplitude of the accuracy difference throughout the

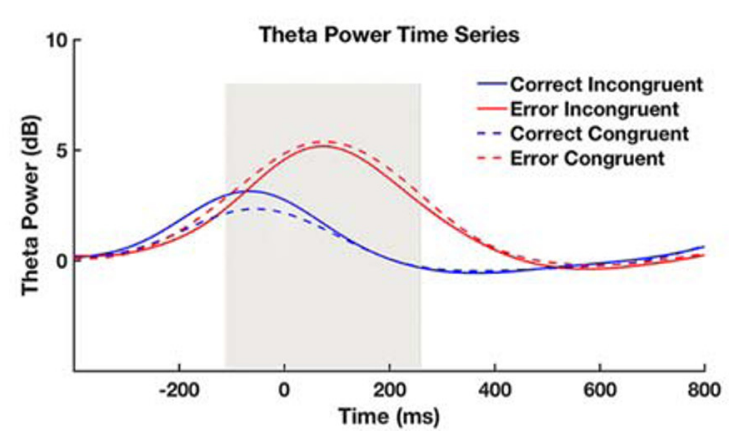

respective analysis window. $\mathbf{b}$ Non-phase-locked theta power at electrode $\mathrm{Cz}$ (collapsed across RSI). The topographic plot signifies the power of the accuracy difference throughout the respective analysis window. Although the epoch time range for time frequency analyses was from $-1,000$ to $1,500 \mathrm{~ms}$, the data were ultimately plotted from -400 to $800 \mathrm{~ms}$ to match the time range of the ERP plot. (Color figure online) 
theta $(z=4.408$, estimate $=.137, S E=.031, p<.001)$, in which larger theta magnitude predicted increased accuracy on the following trial. All other effects regarding theta or ERN failed to reach significance. Therefore, theta explained unique variance in terms of predicting PEA, over and above any effect of the ERN.

\section{Akaike Weights Ratio}

To test which electrophysiological index was a better predictor of post-error behavior, we computed a series of Akaike weight ratios. For PES, the model including the ERN was 1.634 times more likely to be the better model than the one including theta, with a normalized probability of $62.04 \%$ that the model including ERN was preferred to theta. For PEA, the model including theta was 1612.357 times more likely to be the better model than the one including the ERN, with a normalized probability of $99.94 \%$ that the model including theta was the preferred model.

\section{PES PEA mediation model}

When conducting the mediation model for theta and the ERN (see Fig. 5), in order to ensure that the same trials were used for comparing each component to PES and PEA, we lifted the restriction that subsequent trials must be a correct response. In addition, since the mixed-effects models found that the ERN only predicted PES on incongruent trials, the mediation model for the ERN was limited to incongruent errors (however, a separate ERN mediation model that was limited to congruent errors was conducted and did not result in any significant effects; see Supplemental Materials). The mediation analysis for theta revealed a significant total effect $(\beta=$ $.023,95 \%$ CI $[.013, .030], p<.001)$, a significant direct effect of theta on PEA ( $\beta=.021,95 \%$ CI $[.011, .030], p<.001)$, as well as a significant indirect effect of theta on PEA, as mediated by PES ( $\beta=.001,95 \%$ CI $[.0003,0], p=.014)$. For the mediation analysis for the ERN (regarding incongruent errors), there was not a significant total effect $(\beta=-.005,95 \%$ CI $[-.019, .010], p=.480)$, direct effect of ERN on PEA $(\beta=$ $-.003,95 \%$ CI $[-.018, .010], p=.630)$, or indirect effect of ERN on PEA, as mediated by PES $(\beta=-.001,95 \% \mathrm{CI}$ $[-.003,0], p=.120)$.

\section{Discussion}

The present study investigated the neural dynamics of error processing in both the time and frequency domains, as well as associated behavioral phenomena, at the single-trial level. We
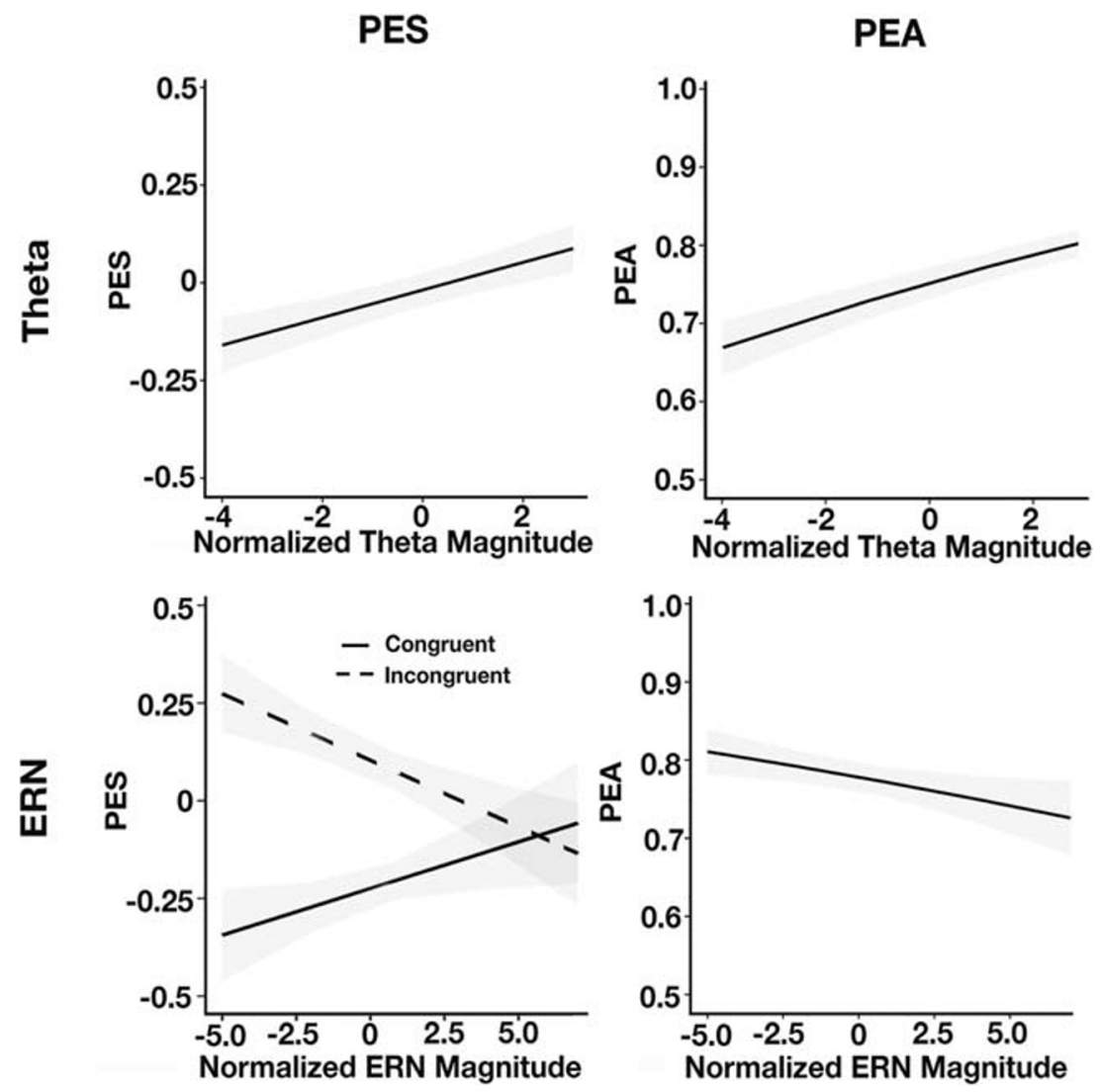

Fig. 4 Single trial models. Displays the single trial models predicting PES and PEA for the ERN and theta. Shaded regions indicate the standard error 
used a technique that enabled us to separately investigate the evoked and induced aspects of the EEG signal (Cohen \& Donner, 2013). We found that at the single-trial level, while the (evoked) ERN was predictive of only PES - and only when errors occurred on incongruent trials-induced frontal midline theta power served as a robust predictor of both PES and PEA, regardless of stimulus congruency. Mediation models of both electrophysiological indices demonstrated that although the relationship between theta and PEA was mediated by PES, there was not a relationship between the ERN and PEA. These data highlight at least partial dissociations in the neural mechanisms indexed by the ERN and induced theta power. Moreover, these data provide evidence for the adaptive theory of PES, when single-trial analyses of induced theta power are also considered.

With regard to the inclusion of RSIs between $200 \mathrm{~ms}$ to $1,200 \mathrm{~ms}$ in the task design, the present study replicated the behavioral findings by Jentzsch and Dudschig (2009), such that short RSIs were associated with increased PES and reduced PEA compared with longer RSIs. Critically, although RSI was included as a factor in the single-trial models, we failed to observe any significant interactions between our electrophysiological measures and RSI. Although future research may wish to further explore the impact that RSI has on various action-monitoring indices, our results suggest the presence of RSI did not inform the relation between our electrophysiological indices and post-error behavior, and are not discussed further (for detailed discussion of the role of RSI in these data, see Beatty et al., 2018).

In concordance with recent work investigating the relation of theta, the ERN, and PES (Kalfaoğlu et al., 2018), the mixed-effects models revealed that both the ERN and induced theta power are capable of predicting PES. More specifically, a larger ERN predicts increased PES (following only incongruent errors), while larger induced theta power predicts

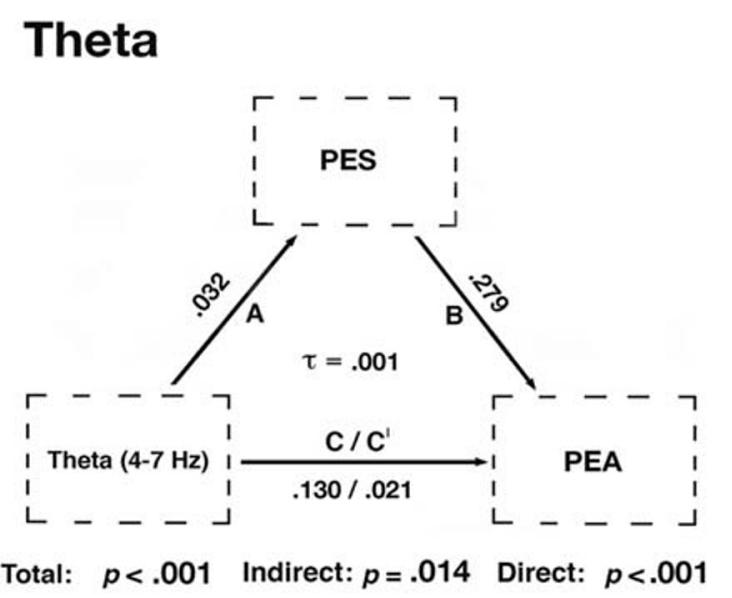

Fig. 5 Mediation models. Displays the mediation models for theta and the ERN, which were centered and scaled in an identical manner as the mixed-effects models. All mediation models limited the current trial to be increased PES, irrespective of stimulus congruency. This provides additional evidence that induced theta power and the evoked ERN index predicts at least partially dissociable neurocognitive processes, perhaps reflecting differing sensitivity to certain aspects of task demands or error types (Maier et al., 2011). According to the Akaike weight ratios, the two neural measures are nearly equivalent in their predictive power for PES, with the ERN providing a marginally better model fit overall. We note that while some studies (the present study included) have observed a relationship between the ERN and PES (Debener et al., 2005; Fischer et al., 2016; Fu et al., 2019; Gehring et al., 1993; Kalfaoğlu et al., 2018; Maier et al., 2011; M. Steinhauser \& Andersen, 2019), there are also some that have not (Beatty et al., 2018; Buzzell, Beatty, et al., 2017; Dudschig \& Jentzsch, 2009; Nieuwenhuis et al., 2001; Valadez \& Simons, 2017; Van der Borght et al., 2016). Although there has been a meta-analysis that investigated the consistency with which the ERN predicts PES (Cavanagh \& Shackman, 2015), this meta-analysis did not investigate relations with PEA. More importantly, the metaanalysis by Cavanagh and Shackman (2015) focused primarily on ERP studies that investigated components thought to be reflective of theta (i.e., the ERN, N2, and FRN). Furthermore, a recent study (Schroder et al., 2019) demonstrated that another explanation for the inconsistency could be the method used to calculate PES, which was not accounted for in the metaanalysis of Cavanagh and Shackman (2015). Another possibility that cannot be ruled out is that the ERN may be more closely associated with error corrections (Rodríguez-Fornells, Kurzbuch, \& Münte, 2002; Ullsperger \& von Cramon, 2006), although we note that there is evidence that the ERN predicts PES irrespective of whether errors were corrected or noncorrected (Kalfaoğlu et al., 2018). Although the current study was not designed to allow for the study of error corrections, future studies should further investigate this possibility.

\section{ERN}

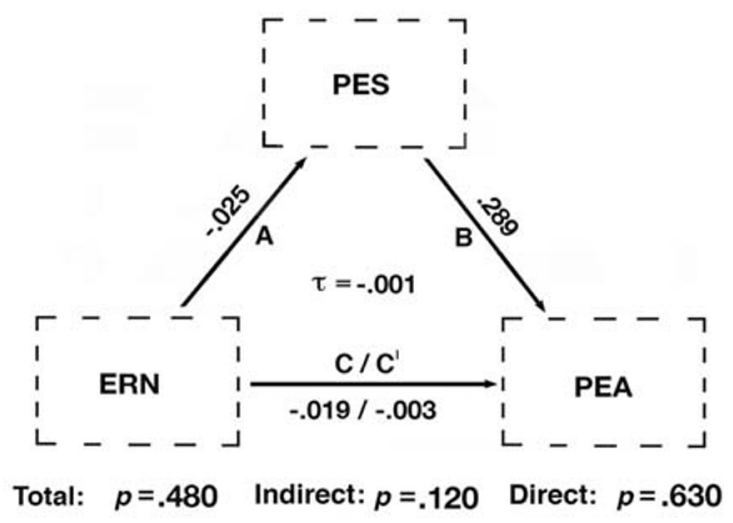

an error trial. $\mathrm{A}, \mathrm{B}$, and $\mathrm{C} / \mathrm{C}^{\prime}$ represent the averaged unstandardized regression coefficients 
In sum, the current study provides additional support that both theta power and the ERN are predictive of PES, although the ERN only predicted PES following incongruent errors.

When investigating whether induced theta power or the ERN predicted PEA, the mixed-effects models, Akaike weight ratio comparison, and mediation models revealed that induced theta power, but not the ERN, predicted PEA. More specifically, we observed that while the positive relationship between theta and PEA was mediated by PES, there were not total, direct, or indirect relationships for the ERN and PEA. Our findings suggest that as the magnitude of error processing increases on the current trial, as indexed by theta but not the ERN, responding on the following post-error trial will be slowed (potentially reflecting increased response caution), resulting in a relative improvement in task performance. These results are consistent with adaptive theories of PES, and moreover, implicate theta power - and not the ERN-in adaptive PES. Therefore, in order to isolate adaptive forms of PES, it is critical to consider theta power and behavior at the single-trial level. Collectively, these results highlight the dissociation between behavioral and electrophysiological indices of action monitoring and the recruitment of cognitive control.

A critical difference in predictive power for theta and the ERN is that only theta acts as a robust predictor of both PES and PEA. Moreover, with regard to the single-trial models that incorporated both theta and the ERN in the same model, theta explained unique variance in terms of predicting next trial behavior, over and above the ERN. Interestingly, despite theta being modulated as a function of congruency at the group level, its ability to predict post-error behavior at the singletrial level was not influenced by stimulus congruency. This is in contrast to the ERN, which was modulated by congruency at the group level, as well as with regard to its ability to predict post-error slowing at the single-trial level. Since both neural indices are sensitive to stimulus congruency at the group level, we propose that the present data can at least partially be explained within the theoretical framework of conflictmonitoring theory (Botvinick et al., 2001; Yeung, Botvinick, $\&$ Cohen, 2004). However, we note that conflict-monitoring theory cannot explain the complete pattern of results for both the ERN and theta. In particular, conflict monitoring theory predicts that, following a response, conflict is highest for congruent errors as opposed to incongruent errors (note that conflict monitoring predicts the opposite prior to the response; see Fig. 4 in Yeung et al., 2004). Our results for theta are entirely consistent with the predictions of conflict monitoring theory (note the similarity between Fig. $3 \mathrm{~b}$ in our paper and the predictions of the conflict monitoring model in Fig. 4 in Yeung et al., 2004); similarly, prior work has linked theta power to conflict specifically (Cohen \& Donner, 2013). However, our results for the ERN, in which the ERN was observed to be larger for incongruent, compared with congruent, errors, cannot be fully explained within the framework of conflict monitoring theory. Given such a discrepancy, we suggest that the ERN data are consistent with a recently proposed extension to conflict monitoring theory, known as the "affective signaling hypothesis" (Dignath, Eder, Steinhauser, \& Kiesel, 2020), which suggests the ERN is sensitive to both conflict and affective aspects of error commission. This work builds on a series of studies by Maier, Steinhauser, and colleagues (Maier, Ernst, \& Steinhauser, 2019; Maier \& Steinhauser, 2016; Maier, Steinhauser, \& Hübner, 2008; Maier et al., 2011), which suggests that negative affect might be associated with some types of errors more than others; in particular, errors occurring in response to incongruent stimuli may be more likely to contain an affective component. Note that such an interpretation is also in line with recent work identifying ventral-frontal sources for the ERN (Buzzell, Richards, et al., 2017). Such theoretical perspectives could explain why we observed the ERN to be larger for incongruent as opposed to congruent trials, and, moreover, why the ERN was only predictive of PES following incongruent errors. We therefore suggest that while theta more closely tracks conflict monitoring, directly in line with the predictions of conflict monitoring theory (Yeung et al., 2004), the ERN arises from a combination of conflict monitoring and additional affective signaling (Dignath et al., 2020). However, additional computational modeling work is needed to direct test such hypotheses.

In conclusion, our data are consistent with the notion that although action-monitoring ERPs and frontal midline theta index functionally related underlying cognitive processes, they are not simply the same process manifested in different domains. Induced frontal midline theta power plays an integral role in action-monitoring processes and appears to more closely relate to adaptive forms of post-error behavior, as compared with the ERN. Finally, these data highlight the importance of simultaneously studying action-monitoring processes in both the neural and behavioral domains at the single-trial level.

\section{References}

Barton, K. (2019). MuMIn: Multi-model inference [Computer software]. Retrieved from http://r-forge.r-project.org/projects/mumin/

Bates, D., Mächler, M., Bolker, B., \& Walker, S. (2014). Fitting linear mixed-effects models using lme4. ArXiv:1406.5823 [Stat]. Retrieved from http://arxiv.org/abs/1406.5823

Beatty, P. J., Buzzell, G. A., Roberts, D. M., \& McDonald, C. G. (2018). Speeded response errors and the error-related negativity modulate early sensory processing. NeuroImage, 183, 112-120. https://doi. org/10.1016/j.neuroimage.2018.08.009

Botvinick, M. M., Braver, T. S., Barch, D. M., Carter, C. S., \& Cohen, J. D. (2001). Conflict monitoring and cognitive control. Psychological Review, 108(3), 624-652. 
Brainard, D. H. (1997). The Psychophysics Toolbox. Spatial Vision, 10(4), 433-436.

Burnham, K. P., \& Anderson, D. R. (2003). Model selection and multimodel inference: A practical information-theoretic approach (2nd ed.). New York, NY: Springer-Verlag.

Buzzell, G. A., Beatty, P. J., Paquette, N. A., Roberts, D. M., \& McDonald, C. G. (2017). Error-induced blindness: Error detection leads to impaired sensory processing and lower accuracy at short response-stimulus intervals. Journal of Neuroscience, 37(11), 2895-2903. https://doi.org/10.1523/JNEUROSCI.1202-16.2017

Buzzell, G. A., Richards, J. E., White, L. K., Barker, T. V., Pine, D. S., \& Fox, N. A. (2017). Development of the error-monitoring system from ages 9-35: Unique insight provided by MRI-constrained source localization of EEG. NeuroImage, 157, 13-26. https://doi. org/10.1016/j.neuroimage.2017.05.045

Cavanagh, J. F., \& Frank, M. J. (2014). Frontal theta as a mechanism for cognitive control. Trends in Cognitive Sciences, 18(8), 414-421. https://doi.org/10.1016/j.tics.2014.04.012

Cavanagh, J. F., \& Shackman, A. J. (2015). Frontal midline theta reflects anxiety and cognitive control: Meta-analytic evidence. Journal of Physiology, Paris, 109(1/3), 3-15. https://doi.org/10.1016/j. jphysparis.2014.04.003

Cohen, M. X. (2014). A neural microcircuit for cognitive conflict detection and signaling. Trends in Neurosciences, 37(9), 480-490. https:// doi.org/10.1016/j.tins.2014.06.004

Cohen, M. X., \& Donner, T. H. (2013). Midfrontal conflict-related thetaband power reflects neural oscillations that predict behavior. Journal of Neurophysiology, 110(12), 2752-2763. https://doi.org/10.1152/ jn. 00479.2013

Debener, S., Ullsperger, M., Siegel, M., Fiehler, K., von Cramon, D. Y., \& Engel, A. K. (2005). Trial-by-trial coupling of concurrent electroencephalogram and functional magnetic resonance imaging identifies the dynamics of performance monitoring. Journal of Neuroscience, 25(50), 11730-11737. https://doi.org/10.1523/JNEUROSCI.328605.2005

Delorme, A., \& Makeig, S. (2004). EEGLAB: An open source toolbox for analysis of single-trial EEG dynamics including independent component analysis. Journal of Neuroscience Methods, 134, 9-21.

Dignath, D., Eder, A. B., Steinhauser, M., \& Kiesel, A. (2020). Conflict monitoring and the affective-signaling hypothesis - An integrative review. Psychonomic Bulletin \& Review. https://doi.org/10.3758/ s13423-019-01668-9

Dudschig, C., \& Jentzsch, I. (2009). Speeding before and slowing after errors: Is it all just strategy? Brain Research, 1296, 56-62. https:// doi.org/10.1016/j.brainres.2009.08.009

Falkenstein, M., Hohnsbein, J., Hoormann, J., \& Blanke, L. (1991). Effects of crossmodal divided attention on late ERP components: II. Error processing in choice reaction tasks. Electroencephalography and Clinical Neurophysiology, 78(6), 447-455.

Fischer, A. G., Danielmeier, C., Villringer, A., Klein, T. A., \& Ullsperger, M. (2016). Gender influences on brain responses to errors and posterror adjustments. Scientific Reports, 6(1), 1-11. https://doi. org/10.1038/srep24435

Fu, Z., Wu, D.-A. J., Ross, I., Chung, J. M., Mamelak, A. N., Adolphs, R., \& Rutishauser, U. (2019). Single-neuron correlates of error monitoring and posterror adjustments in human medial frontal cortex. Neuron, 101(1), 165-177.e5. https://doi.org/10.1016/j.neuron. 2018.11.016

Gehring, W. J., Goss, B., Coles, M. G. H., Meyer, D. E., \& Donchin, E. (1993). A neural system for error detection and compensation. Psychological Science, 4(6), 385-390. https://doi.org/10.1111/j. 1467-9280.1993.tb00586.x

Gelman, A., \& Stern, H. (2006). The difference between "significant" and "not significant" is not itself statistically significant. The American
Statistician, 60(4), 328-331. https://doi.org/10.1198/ 000313006 X152649

Hassall, C. D., McDonald, C. G., \& Krigolson, O. E. (2019). Ready, set, explore! Event-related potentials reveal the time-course of exploratory decisions. Brain Research, 1719, 183-193. https://doi.org/10. 1016/j.brainres.2019.05.039

Imai, K., Keele, L. J., \& Tingley, D. (2010). A general approach to causal mediation analysis. Psychological Methods, 15(4), 309-334. https:// doi.org/10.1037/a0020761

Jentzsch, I., \& Dudschig, C. (2009). Why do we slow down after an error? Mechanisms underlying the effects of posterror slowing. The Quarterly Journal of Experimental Psychology, 62(2), 209218. https://doi.org/10.1080/17470210802240655

Kalfaoğlu, Ç., Stafford, T., \& Milne, E. (2018). Frontal theta band oscillations predict error correction and posterror slowing in typing. Journal of Experimental Psychology: Human Perception and Performance, 44(1), 69. https://doi.org/10.1037/xhp0000417

Kayser, J., \& Tenke, C. E. (2006). Principal components analysis of Laplacian waveforms as a generic method for identifying ERP generator patterns: II. Adequacy of low-density estimates. Clinical Neurophysiology, 117(2), 369-380. https://doi.org/10.1016/j. clinph.2005.08.033

King, J. A., Korb, F. M., von Cramon, D. Y., \& Ullsperger, M. (2010). Posterror behavioral adjustments are facilitated by activation and suppression of task-relevant and task-irrelevant information processing. Journal of Neuroscience, 30(38), 12759-12769. https://doi.org/ 10.1523/JNEUROSCI.3274-10.2010

Kleiner, M., Brainard, D. H., Pelli, D., Ingling, A., Murray, R., \& Broussard, C. (2007). What's new in Psychtoolbox-3. Perception, 36, 1-16. https://doi.org/10.1068/v070821

Kuznetsova, A., Brockhoff, P. B., \& Christensen, R. H. B. (2016). Package "ImerTest" (R Package Version, 2) [Computer software]. Retrieved from http://cran.uib.no/web/packages/lmerTest/lmerTest. pdf

Laming, D. (1979). Choice reaction performance following an error. Acta Psychologica, 43(3), 199-224. https://doi.org/10.1016/00016918(79)90026-X

Lopez-Calderon, J., \& Luck, S. J. (2014). ERPLAB: An open-source toolbox for the analysis of event-related potentials. Frontiers in Human Neuroscience, 8. https://doi.org/10.3389/fnhum.2014. 00213

Luu, P., \& Tucker, D. M. (2001). Regulating action: Alternating activation of midline frontal and motor cortical networks. Clinical Neurophysiology: Official Journal of the International Federation of Clinical Neurophysiology, 112(7), 1295-1306.

Luu, P., Tucker, D. M., \& Makeig, S. (2004). Frontal midline theta and the error-related negativity: Neurophysiological mechanisms of action regulation. Clinical Neurophysiology: Official Journal of the International Federation of Clinical Neurophysiology, 115(8), 1821-1835. https://doi.org/10.1016/j.clinph.2004.03.031

Maier, M., Steinhauser, M., \& Hübner, R. (2008). Is the error-related negativity amplitude related to error detectability? Evidence from effects of different error types. Journal of Cognitive Neuroscience, 20(12), 2263-2273. https://doi.org/10.1162/jocn.2008.20159

Maier, M. E., Ernst, B., \& Steinhauser, M. (2019). Error-related pupil dilation is sensitive to the evaluation of different error types. Biological Psychology, 141, 25-34. https://doi.org/10.1016/j. biopsycho.2018.12.013

Maier, M. E., \& Steinhauser, M. (2016). Error significance but not error expectancy predicts error-related negativities for different error types. Behavioural Brain Research SreeTestContent1, 297, 259267. https://doi.org/10.1016/j.bbr.2015.10.031

Maier, M. E., Yeung, N., \& Steinhauser, M. (2011). Error-related brain activity and adjustments of selective attention following errors. NeuroImage, 56(4), 2339-2347. https://doi.org/10.1016/j. neuroimage.2011.03.083 
Marco-Pallarés, J., Camara, E., Münte, T. F., \& Rodríguez-Fornells, A. (2008). Neural mechanisms underlying adaptive actions after slips. Journal of Cognitive Neuroscience, 20(9), 1595-1610. https://doi. org/10.1162/jocn.2008.20117

Nieuwenhuis, S., Forstmann, B. U., \& Wagenmakers, E.-J. (2011). Erroneous analyses of interactions in neuroscience: A problem of significance. Nature Neuroscience, 14(9), 1105-1107. https://doi. org $110.1038 / \mathrm{nn} .2886$

Nieuwenhuis, S., Ridderinkhof, K. R., Blom, J., Band, G. P. H., \& Kok, A. (2001). Error-related brain potentials are differentially related to awareness of response errors: Evidence from an antisaccade task. Psychophysiology, 38(5), 752-760. https://doi.org/10.1111/14698986.3850752

Notebaert, W., Houtman, F., Opstal, F. V., Gevers, W., Fias, W., \& Verguts, T. (2009). Posterror slowing: An orienting account. Cognition, 111(2), 275-279. https://doi.org/10.1016/j.cognition. 2009.02.002

Olvet, D. M., \& Hajcak, G. (2009). The stability of error-related brain activity with increasing trials. Psychophysiology, 46(5), 957-961. https://doi.org/10.1111/j.1469-8986.2009.00848.x

Pelli, D. G. (1997). The VideoToolbox software for visual psychophysics: Transforming numbers into movies. Spatial Vision, 10(4), 437-442.

Purcell, B. A., \& Kiani, R. (2016). Neural mechanisms of posterror adjustments of decision policy in parietal cortex. Neuron, 89(3), 658671. https://doi.org/10.1016/j.neuron.2015.12.027

Rodríguez-Fornells, A., Kurzbuch, A. R., \& Münte, T. F. (2002). Time course of error detection and correction in humans: Neurophysiological evidence. Journal of Neuroscience, 22(22), 9990-9996.

Schroder, H. S., Nickels, S., Cardenas, E., Breiger, M., Perlo, S., \& Pizzagalli, D. A. (2019). Optimizing assessments of posterror slowing: A neurobehavioral investigation of a flanker task. Psychophysiology, 57(2), e13473. https://doi.org/10.1111/psyp. 13473

Steinhauser, M., \& Andersen, S. K. (2019). Rapid adaptive adjustments of selective attention following errors revealed by the time course of steady-state visual evoked potentials. NeuroImage, 186, 83-92. https://doi.org/10.1016/j.neuroimage.2018.10.059

Steinhauser, R., Wirth, R., Kunde, W., Janczyk, M., \& Steinhauser, M. (2018). Common mechanisms in error monitoring and action effect monitoring. Cognitive, Affective, \& Behavioral Neuroscience, 18(6), 1159-1171. https://doi.org/10.3758/s13415-018-0628-y

Tingley, D., Yamamoto, T., Hirose, K., Keele, L., \& Imai, K. (2014). mediation: R Package for causal mediation analysis. Journal of Statistical Software, 59(1), 1-38. https://doi.org/10.18637/jss.v059. i05

Trujillo, L. T., \& Allen, J. J. B. (2007). Theta EEG dynamics of the errorrelated negativity. Clinical Neurophysiology, 118(3), 645-668. https://doi.org/10.1016/j.clinph.2006.11.009

Ullsperger, M., \& Danielmeier, C. (2016). Reducing speed and sight: How adaptive is posterror slowing? Neuron, 89(3), 430-432. https://doi.org/10.1016/j.neuron.2016.01.035

Ullsperger, M., Fischer, A. G., Nigbur, R., \& Endrass, T. (2014). Neural mechanisms and temporal dynamics of performance monitoring. Trends in Cognitive Sciences, 18(5), 259-267. https://doi.org/10. 1016/j.tics.2014.02.009
Ullsperger, M., \& von Cramon, D. Y. (2006). How does error correction differ from error signaling? An event-related potential study. Brain Research, 1105(1), 102-109. https://doi.org/10.1016/j.brainres. 2006.01.007

Valadez, E. A., \& Simons, R. F. (2017). The power of frontal midline theta and post-error slowing to predict performance recovery: Evidence for compensatory mechanisms. Psychophysiology, 55(4), e13010. https://doi.org/10.1111/psyp.13010

van den Brink, R. L., Wynn, S. C., \& Nieuwenhuis, S. (2014). Posterror slowing as a consequence of disturbed low-frequency oscillatory phase entrainment. Journal of Neuroscience, 34(33), 1109611105. https://doi.org/10.1523/JNEUROSCI.4991-13.2014

Van der Borght, L., Schevernels, H., Burle, B., \& Notebaert, W. (2016). Errors disrupt subsequent early attentional processes. PLOS ONE, 11(4), e0151843. https://doi.org/10.1371/journal.pone.0151843

Wagenmakers, E.-J., \& Farrell, S. (2004). AIC model selection using Akaike weights. Psychonomic Bulletin \& Review, 11(1), 192-196. https://doi.org/10.3758/BF03206482

Wessel, J. R. (2017). An adaptive orienting theory of error processing. Psychophysiology, 53(3), e13041. https://doi.org/10.1111/psyp. 13041

Wessel, J. R., \& Aron, A. R. (2017). On the globality of motor suppression: Unexpected events and their influence on behavior and cognition. Neuron, 93(2), 259-280. https://doi.org/10.1016/j.neuron. 2016.12.013

Winkler, I., Debener, S., Müller, K. R., \& Tangermann, M. (2015). On the influence of high-pass filtering on ICA-based artifact reduction in EEG-ERP. Proceedings of the 37th Annual International Conference of the IEEE Engineering in Medicine and Biology Society (EMBC) (pp. 4101-4105). https://doi.org/10.1109/EMBC. 2015.7319296

Yeung, N., Bogacz, R., Holroyd, C. B., \& Cohen, J. D. (2004). Detection of synchronized oscillations in the electroencephalogram: An evaluation of methods. Psychophysiology, 41(6), 822-832. https://doi. org/10.1111/j.0048-5772.2004.00239.x

Yeung, N., Bogacz, R., Holroyd, C. B., Nieuwenhuis, S., \& Cohen, J. D. (2007). Theta phase resetting and the error-related negativity. Psychophysiology, 44(1), 39-49. https://doi.org/10.1111/j.14698986.2006.00482.x

Yeung, N., Botvinick, M. M., \& Cohen, J. D. (2004). The neural basis of error detection: Conflict monitoring and the error-related negativity. Psychological Review, 111(4), 931-959. https://doi.org/10.1037/ 0033-295X.111.4.931

Open practices statement None of the data or materials for the experiments reported here is available, and none of the experiments was preregistered.

Publisher's note Springer Nature remains neutral with regard to jurisdictional claims in published maps and institutional affiliations. 